\title{
Influence of refractive index matching on the photon diffuse reflectance
}

\author{
D Y Churmakov, I V Meglinski and D A Greenhalgh \\ School of Engineering, University of Cranfield, Cranfield, MK43 OAL, UK \\ E-mail: i.meglinski@cranfield.ac.uk
}

\begin{abstract}
Photon migration in a randomly inhomogeneous, highly scattering and absorbing semi-infinite medium with a plane boundary is considered by a Monte Carlo (MC) technique. The employed MC technique combines the statistical weight scheme and real photon paths simulation, allowing the exclusion of the energy conservation problem. The internal reflection of the scattered radiation on the medium interface is taken into account by allowing the trajectories of photon packets to be split into reflected and transmitted parts. The spatial photon sensitivity profile (SPSP), spatially resolved diffuse reflectance and angular and spatial photon detector weight distributions are considered in terms of Fresnel's reflection/refraction on the boundary of the medium. The effect of the refractive index match is predicted correctly by the MC method and by the diffusion approximation. The results demonstrate that matching of the refractive index of the medium significantly improves the contrast and spatial resolution of the spatial photon sensitivity profile (SPSP). The results of simulation of the spatially resolved diffuse reflectance agree well with the results predicted by the diffusion approximation and the experimental results reported earlier.
\end{abstract}

\section{Introduction}

Propagation of laser radiation in randomly inhomogeneous, highly scattering turbid media has been intensively researched internationally for the last few decades (Wiersma et al 1997). The continuing interest in this problem is explained by a number of applications including life science. The common problem in optical diagnostics and tomography is that optical radiation travels great distances in the tissues due to multiple scattering. This reason and the complex multi-component structure of tissues lead to difficulties in the quantification of optical reflectance spectroscopy (Kienle et al 1996, Mourant 1997, Meglinsky and Matcher 2001a, Matcher 2002) and poor resolution and contrast in optical tomography and image 
reconstruction techniques (Arridge 1995, Okada 2000, Jermy and Allen 2002). This requires an understanding of the spatial distribution of the detector depth sensitivity profile inside the medium, or the so-called sampling volume.

Since the direct measurements of the sampling volume cannot be obtained experimentally, various mathematical methods have evolved (Nossal et al 1988, Hiraoka et al 1993, Arridge 1995, Meglinsky and Matcher 2001a). Theoretical methods of light propagation are typically based on the transport theory or the diffusion approximation (Ishimaru 1978). In the framework of this theory the effects of refraction/reflection on the medium boundary are significant, because of the mismatch of the refractive indices. This leads to increasing photon flight time due to the internal reflection (Zhu et al 1991). Various approaches to include mismatched boundary conditions have been developed (Lagendjik et al 1989, Zhu et al 1991, Haskell et al 1994, Star 1995, Kienle and Patterson 1997, Ripoll and Nieto-Vesperinas 1999).

Recently, Farrell and Patterson (2001) demonstrated that matching of the refractive indices of the exterior and phantom media caused decrease of the internal fluence rate. This accounted for reduction of the internal reflection effects on the medium surface. They also considered the changes in spatially resolved diffuse reflectance due to medium refractive index matching.

Other recent studies aimed at a concurrent improvement of penetration depth and imaging contrast for a more effective optical diagnostics have been carried out (Tuchin et al 1997, Vargas et al 1999, Tuchin 2000, Meglinski et al 2001, Vargas et al 2001, Wang et al 2001). They conclude that matching of the refractive indices of the structural elements of tissue cells occurs. This produces a temporal effect of light scatter reduction that increases the transparency of the upper skin layers and, consequently, allows unrestricted light to permeate deeper into tissues.

However, it is difficult to distinguish the separate impact of the medium scattering reduction and the refractive index match on the imaging contrast improvement, especially when reduction of scattering of the medium significantly prevails over the effect of exterior medium/medium refractive indices match.

In this paper we consider the influence of the refractive index match at the interface of homogeneous semi-infinite highly scattering media on the spatial photon sensitivity profile (SPSP) and intensity diffuse reflectance. A set of the computational steps of the MC model is revised in section 2. The results of the simulation including spatial/angular photon weight distribution and SPSPs observed in respect of the Fresnel reflection are presented and discussed in section 3. The results of the spatially resolved diffuse reflectance simulation are compared with the results predicted by the diffusion theory. Finally, summaries and conclusions of the study are given in section 4 .

\section{Monte Carlo technique}

The stochastic numerical Monte Carlo (MC) technique has many advantages over analytical models: various boundary conditions can be taken into account, the technique allows looking at many different physical quantities, the method is suitable for tackling the difficulties of light transport in complex geometry, including 3D modelling, etc. There are a great number of MC techniques (Sobol' 1975, Witt 1977, Meier et al 1978, Wilson and Adam 1983, Bonner et al 1987, Keijzer et al 1989, Yaroslavsky and Tuchin 1992, Wang et al 1995, Okada and Firbank 1997, Meglinsky and Matcher 2001a, Jermy and Allen 2002). However, these MC techniques have been developed for specific objectives and various applications.

Wilson and Adam (1983), Keijzer et al (1989), Prahl et al (1989), Yaroslavsky and Tuchin (1992) and Wang et al (1995) developed the MC method for the dosimetry of laser radiation in biological tissues. The peculiarity of these models is based on the photon weight variance 
reduction. This so-called implicit capture technique (Witt 1977) is equivalent to modelling the photon propagation along each possible pathway within the medium as a group of photons (photon packet). This is attained by attributing the initial weight $W_{\text {in }}$ to all the photons. This weight is attenuated exponentially due to photon random walk in a multiple scattering and absorbing medium. When the weight becomes too small, the technique, known as 'Russian roulette', gives each photon a chance in $m$ of surviving with a weight $m W$. Otherwise, it terminates the further packet propagation in the medium and generates absorption of the photon weight. This distinguished scheme intends to balance incident radiation with the radiation absorbed and scattered within the medium. However, assigning of additional weight to a photon packet results in an unjustified increase of its pathlength in the medium, which consequently affects the distribution of the photon paths and gives the uncertainty to the method. The physical interpretation is also complicated and inconsistent.

In the so-called 'random walk' model (Bonner et al 1987, Gandjbakhche and Weiss 1995, Chernomordik et al 1999), the kinetics of the photon migration is evaluated numerically within the cubic lattice by a fixed step equalling the lattice scale. The Lambert-Beer law describes the absorption between the nodes of the lattice. For the sake of simplicity, internal interfacial boundaries are assumed to be perfectly plain. However, if the medium surface or the interfaces of the internal boundaries of the layers (or inhomogeneities) are complex and/or randomly wavy, difficulties issue from the presence of refractive index mismatch and cubic grating of the medium.

The approach recently reported by Jermy and Allen $(2001,2002)$ consists of dividing the medium on a cubic grid, with the consequent tracking of individual photons along their trajectories. In this MC scheme a photon trajectory depends on the density of the scattering centres in a voxel, their scattering cross sections and the probability density function of the angular deviation of the photon trajectory. This algorithm is applied for imaging of the photon migration in low-order multiple scattering media, and is potentially appealing for the dynamic light scattering. It is optimized to handle inhomogeneous media with three-dimensional structures such as sprays. However, in the case of high density of the scatters in voxels, this technique is equivalent to the MC scheme mentioned below (Meier et al 1978, Okada 2000, Meglinskii and Matcher 2001b, Meglinski and Matcher 2002).

We employed the MC technique, which combines the statistical weight scheme and effective optical photon paths simulation (Meglinsky and Matcher 2001a, Meglinskii and Matcher 2001b). This approach allows exclusion of the energy conservation problem which occurs in the 'roulette' method, and interpret results avoiding additional calculations. Below we describe the main peculiarities and distinctions of this code.

The simulation is based on the modelling of a large number of possible trajectories of the photon packets from the site of photon injection into the medium (source) to the site where the photons leave the medium (detector) (figure 1). Modelling of an individual photon packet trajectory consists of the sequence of the following elementary simulations: photon pathlength generation, scattering events and reflection/refraction on the medium boundary. The initial and final states of the photons are entirely determined by the source and the detector geometries and numerical apertures.

The photon pathlength $l$ can take on any positive value with the probability density function (Sobol' 1975):

$p(l)=\mu_{t} \exp \left(-\mu_{t} l\right)=\mu_{s} \exp \left(-\mu_{s} l\right) \exp \left(-\mu_{a} l\right)+\mu_{a} \exp \left(-\mu_{a} l\right) \exp \left(-\mu_{s} l\right)$

where $\mu_{t}=\mu_{s}+\mu_{a}$ is the extinction coefficient, $\mu_{s}$ and $\mu_{a}$ are the scattering and absorption coefficients, respectively. The augend in (1) describes the probability of the photon scattering, and its reduction due to absorption. Respectively, the addend determines the probability 


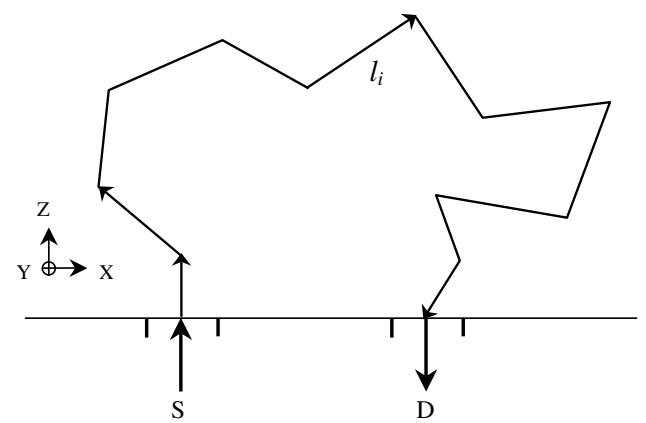

Figure 1. We consider a semi-infinite randomly inhomogeneous medium with a planar interface, as the most common geometry inherent to biological tissues. $S$ and $D$ are the areas of source and detector, respectively. $l_{i}$ is the photon packet pathlength between the $i$ th and $(i+1)$ th scattering events. The scattering events are determined according to the density of the scattering particles described by the scattering coefficient $\mu_{s}$. The absorption coefficient $\mu_{a}$, the anisotropy factor $g$ and the refractive index $n$ describe the other optical properties of the medium.

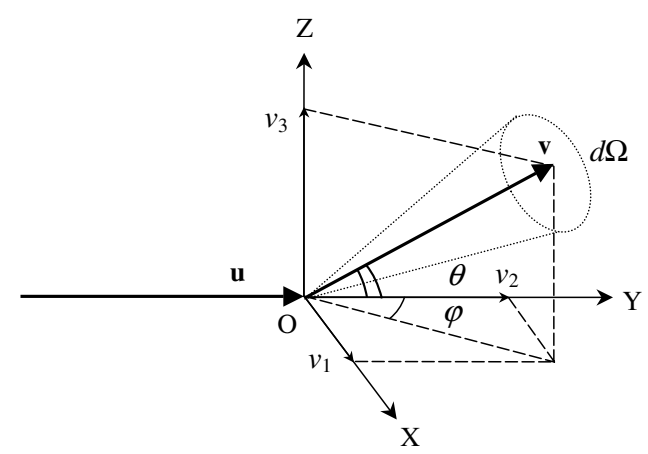

Figure 2. The Cartesian coordinate system $(X Y Z)$ is employed to specify the position of a photon in the modelling medium: $\boldsymbol{u}$ and $\boldsymbol{v}$ are the directional unit vectors lying along the initial direction of a photon packet propagation and a new photon packet direction, respectively. $\theta$ and $\phi$ are the polar and azimuthal angles of deviation from the initial photon direction $\boldsymbol{u}$. $\mathrm{d} \Omega$ is the elementary solid angle, and $v_{1}, v_{2}, v_{3}$ are the directional cosines.

of the photon absorption in the medium reduced by the scattering. In terms of optical diagnostics, however, the detected photons are mainly of interest rather than the photons lost and absorbed in the medium. Assuming the independence of the absorption and scattering in the medium, we denote the photon pathlength probability density function (1) by the augend only. This allows stimulating the scattering and absorption of the photon packets in a medium separately. Therefore, the pathlength that a photon packet moves at the $i$ th step (figure 1) is modelled as

$$
l_{i}=-\frac{\ln (\xi)}{\mu_{s}}
$$

where $\xi$ is a uniformly distributed random number between 0 and 1 . The Cartesian coordinate system $(X Y Z)$ is employed to specify the position of a photon in the modelling medium. Except $x, y, z$ coordinates, each step of the photon packet motion in a medium is specified by polar $\theta$ and azimuthal $\varphi$ angles (see figure 2). These angles describe the scattering of 
the photon packets, characterized by a scattering phase function, and defined as normalized angular scattering cross section (Thomas and Stamnes 1999):

$$
p(\boldsymbol{u}, \boldsymbol{v}) \equiv \frac{\sigma(\boldsymbol{u}, \boldsymbol{v})}{\int_{4 \pi} \mathrm{d} \Omega \sigma(\boldsymbol{u}, \boldsymbol{v})}\left[\mathrm{sr}^{-1}\right] .
$$

Here $\boldsymbol{u}$ and $\boldsymbol{v}$ are the photon packet directions before and after the scattering, respectively (see figure 2 for details), $\sigma$ is the angular cross section $\left(\mathrm{m}^{-1} \mathrm{sr}^{-1}\right)$, and $\mathrm{d} \Omega$ is the elementary solid angle. Following earlier works (Flock et al 1989, Keijzer et al 1989, Yaroslavsky and Tuchin 1992, Wang et al 1995), we use homogeneous phase function, relative to the mutual orientation of vectors $\boldsymbol{u}$ and $\boldsymbol{v}$. This function is known as a Henyey-Greenstein scattering phase function (Henyey and Greenstein 1941):

$$
p(\boldsymbol{u}, \boldsymbol{v})=p(\cos (\theta))=\frac{1}{4 \pi} \frac{\left(1-g^{2}\right)}{\left(1+g^{2}-2 g \cos \theta\right)^{3 / 2}} .
$$

To make the progress of coordinates smoother we convert $\theta$ and $\varphi$ angles to their corresponding direction cosines. Initially the photons packets are emitted perpendicular to the medium surface, that is in terms of the initial directional cosines $\left(u_{1}, u_{2}, u_{3}\right)$ represented as $u_{1}=0$, $u_{2}=0, u_{3}=1$. New values for the directional cosines, $v_{1}, v_{2}, v_{3}$, are calculated as

$$
\left(\begin{array}{l}
v_{1} \\
v_{2} \\
v_{3}
\end{array}\right)=\left[\begin{array}{ccc}
\frac{1}{\sqrt{1-u_{3}^{2}}} u_{1} u_{3} & -\frac{1}{\sqrt{1-u_{3}^{2}}} u_{2} & u_{1} \\
\frac{1}{\sqrt{1-u_{3}^{2}}} u_{2} u_{3} & \frac{1}{\sqrt{1-u_{3}^{2}}} u_{1} & u_{2} \\
-\sqrt{1-u_{3}^{2}} & 0 & u_{3}
\end{array}\right]\left(\begin{array}{l}
\sin (\theta) \cos (\varphi) \\
\sin (\theta) \sin (\varphi) \\
\cos (\theta)
\end{array}\right) \text {. }
$$

The specular and internal reflections on the medium boundary are taken into account by splitting the photon packet into the reflected and the transmitted parts, whose weights are attenuated as

$$
W=\left(1-R\left(\theta_{i}\right)\right)\left(1-R_{\text {in }}\right) W_{\text {in }}\left[\prod_{p=1}^{M-1} R\left(\theta_{i}\right)\right]
$$

where $W_{\text {in }}$ is the initial weight of the photon packet, $M$ is the number of the photon packet reflections/refractions on the medium boundary and $R_{\text {in }}$ is the Fresnel coefficient relating to the initial reflection $\left(\theta=0^{0}\right)$ on the medium boundary surface, when the photon packet enters the medium. $R\left(\theta_{i}\right)$ is the Fresnel reflection coefficient

$$
R\left(\theta_{i}\right)= \begin{cases}\frac{\left(n-n_{0}\right)^{2}}{\left(n+n_{0}\right)^{2}} & \text { if } \theta_{i}=0^{0} \\ \frac{1}{2}\left[\frac{\sin ^{2}\left(\theta_{i}-\theta_{t}\right)}{\sin ^{2}\left(\theta_{i}+\theta_{t}\right)}+\frac{\tan ^{2}\left(\theta_{i}-\theta_{t}\right)}{\tan ^{2}\left(\theta_{i}+\theta_{t}\right)}\right] & \text { if } 0^{0}<\theta_{i}<\sin ^{-1}\left(\frac{\mathrm{n}_{0}}{\mathrm{n}}\right) \\ 1 & \text { if } \sin ^{-1}\left(\frac{\mathrm{n}_{0}}{\mathrm{n}}\right)<\theta_{i}<90^{0} .\end{cases}
$$

Here $\theta_{i}$ and $\theta_{t}$ are the angles of photon boundary incidence and transmittance, respectively; $n$ and $n_{0}$ describe the refraction coefficients of corresponding and ambient media.

The simulation of a photon packet tracing is stopped when its statistical weight is less than $10^{-3}$, or a photon packet has been scattered more than $10^{4}$ times. The first option means that the weight is too small and the packet no longer contributes to the detected signal, whereas the latter condition is similar to interruption in the photon history progress if it went too far from a detector region. The total number of the detected photon packets (usually $10^{5}-10^{7}$ ) is defined before each simulation as suggested (Kienle et al 1996). The individual trajectory of 


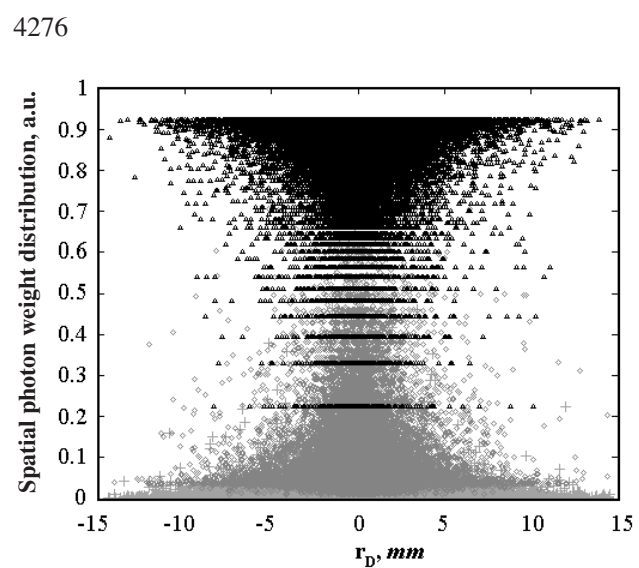

(a)

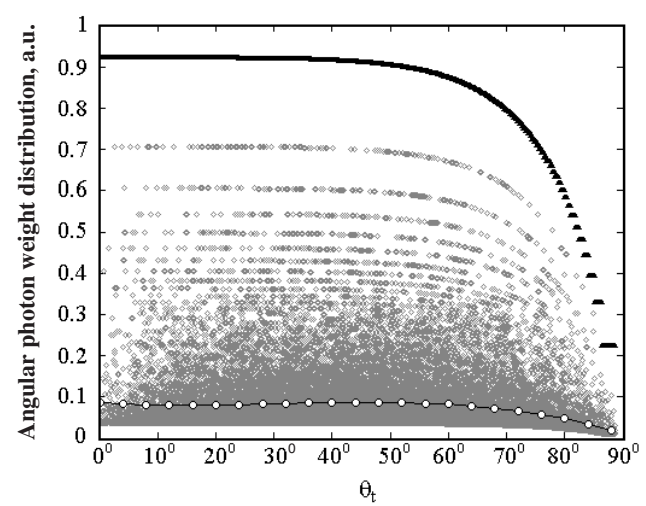

(b)

Figure 3. Photon packets weight distribution on the detector: (a) spatial photon weight distribution versus radius of the detector area $\left(\boldsymbol{r}_{\mathrm{D}}\right)$; (b) angular photon weight distribution over the angle of exit $\theta_{t}$, defined as the angle between the photon packet exiting direction and the outward normal to the medium surface (see figure 4 for details). The triangles $(\Delta)$ specify the weight of the photon packets experienced by one reflection/refraction event only on the medium boundary. Diamonds $(\diamond)$ represent the weight of the photon packets having acted on the medium boundary twice, and circles $(O)$ point out their average angular weight distribution. Crosses $(+)$ show the weight of the high order photon packets reflected/refracted on the medium boundary in (a), and ignored in (b).

each photon packet which arrived at the detector through the non-absorbing medium $\left(\mu_{a}=0\right)$ is stored in a data file.

We include the absorption of the medium by recalculating the statistical weight of each photon packet according to its trajectory, i.e.

$$
W\left(\boldsymbol{r}_{\mathrm{D}}\right)=W_{j} \exp \left(-\sum_{i=1}^{N_{j}} \mu_{a} l_{i}\right) .
$$

Here, $N_{j}$ is the number of scattering events undergone by the $j$ th photon packet during its random walk, $l_{i}$ is the photon packet pathlength at the $i$ th scattering event (2), $W_{j}$ is the statistical weight of the $j$ th photon packet reaching the detector area in the absorption free medium $\left(\mu_{a}=0\right)(6)$. Such an approach of separate simulation of scattering and absorption agrees with the microscopic Beer-Lambert law, and allows the rapid recalculation of the sounding radiation intensity on the detector area $\boldsymbol{r}_{\mathrm{D}}$ for a set of the medium absorption.

\section{Results and discussion}

\subsection{Photon weight distribution on the medium surface}

The results of the simulation of the spatial and angular photon weight distributions at the detector coincident with the medium boundary are shown in figure 3 . We consider the semiinfinite highly scattering medium whose optical properties are: $\mu_{s}=30 \mathrm{~mm}^{-1}, \mu_{a}=0 \mathrm{~mm}^{-1}$, $g=0.9, n=1.5$. The properties of the scatter are chosen as most typical for skin tissues (Tuchin 2000). The refractive index of the ambient medium $n_{0}$ is 1.0. The detector area coincident with the medium surface is limited by a circle of $15 \mathrm{~mm}$ radius, sharing the centre with the pencil radiation source.

In figure 3(a) the axis of the abscissas shows the detected photons coordinates on the detector, while the axis of the ordinates illustrates the weight of the photon packets. The 


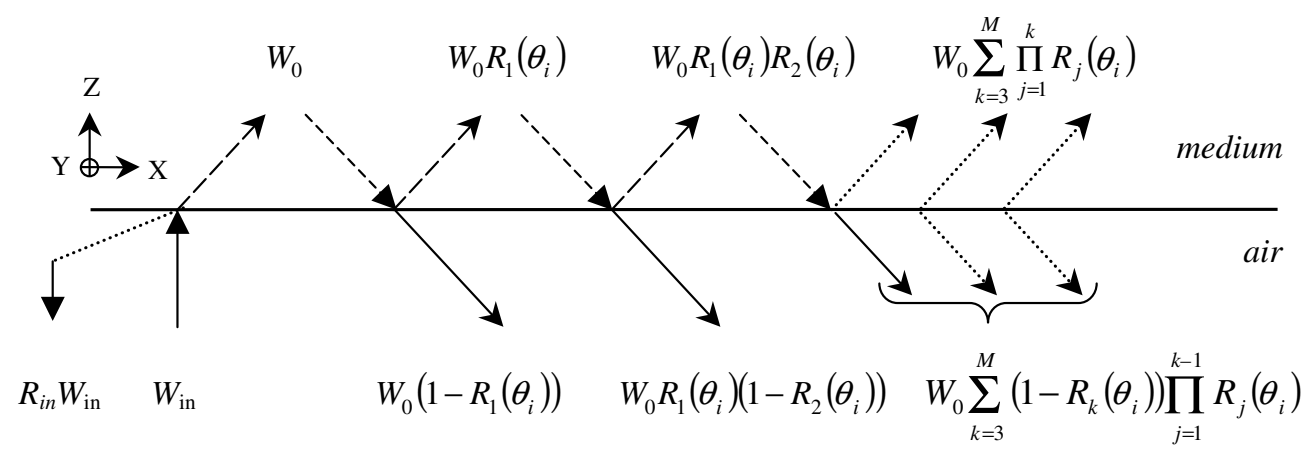

Figure 4. A schematic presentation of the photon packet medium boundary reflection/refraction arrangement of interactions. $W_{\text {in }}$ is the initial weight of the photon packets (normally equal to 1 ), $R_{\text {in }}$ is the Fresnel coefficient of the initial specular reflection. $W_{0}=\left(1-R_{\text {in }}\right) W_{\text {in }}$ is the photon packet weight after initial specular reflection. $\theta_{i}$ and $\theta_{t}$ are the angles of incidence and transmittance (exit), respectively. $R\left(\theta_{i}\right)$ is the Fresnel reflection coefficient. $W_{1}$ shows the fraction of a photon packet detected free of surface reflections/refractions (except possible total internal reflection events). $W_{2}$ is the fraction of the photon packet reflected/refracted once on the medium surface before the detection. $W_{3}$ represents the summarised weight fractions of high order photon packet medium surface interactions.

weight of the photon packets demonstrated by the triangles is diminished due to the Fresnel refraction and reflection on the medium boundary (excluding specular reflection of the initial photon emitted and the total internal reflection):

$$
W_{1}=\left(1-R\left(\theta_{i}\right)\right) W_{0} .
$$

The photon weights remain unchanged between the reflections/refractions at the medium boundary. The schematic illustration of the photon medium boundary interactions is represented in figure 4.

A set of diamonds (see figure 3) shows the weight distribution of those photon packets that have refractively interacted at the medium boundary twice (see figure 4), i.e.

$$
W_{2}=\left(1-R\left(\theta_{i}\right)\right) W_{0} R\left(\theta_{i}\right) .
$$

The weights of the high order photon medium boundary reflection/refraction interactions are

$$
W_{3}=W_{0} \sum_{k=3}^{M}\left(1-R_{k}\left(\theta_{i}\right)\right) \prod_{j=1}^{k-1} R_{j}\left(\theta_{i}\right)
$$

where $M$ is the total number of photon packet boundary interactions.

The angular photon weight distribution $W_{1,2}\left(\theta_{t}\right)$ is shown in figure $3(\mathrm{~b})$ in the same manner. Here, the axis of the abscissas presents the angles of the photon packet medium escaping $\theta_{t}$, and the axis of the ordinates presents the photon packet weight. $W_{3}$ is depicted on figure 3(a) with crosses and ignored on figure 3(b) as quantitatively negligible. It is worth mentioning that the normalized $W_{3}$ photon weight distribution is negligible compare to the $W_{1}$ distribution. Circles (see figure 3(b)) present the average angular $W_{2}$ weight distribution. The angular weight distribution of the photon packets is equivalent to the Fresnel transmission coefficient versus the angle of the photon packet medium escaping $\theta_{t}$ (figure 5).

The discrete structure seen both at the spatial and angular photon weight distributions (see figures 3(a) and (b)) results from the stepwise cosine function calculation. The values of directional cosines are handled as discrete elements of arrays generated in advance of the MC code, as was suggested by Lux and Koblinger (1991). The lookup reference tables for 


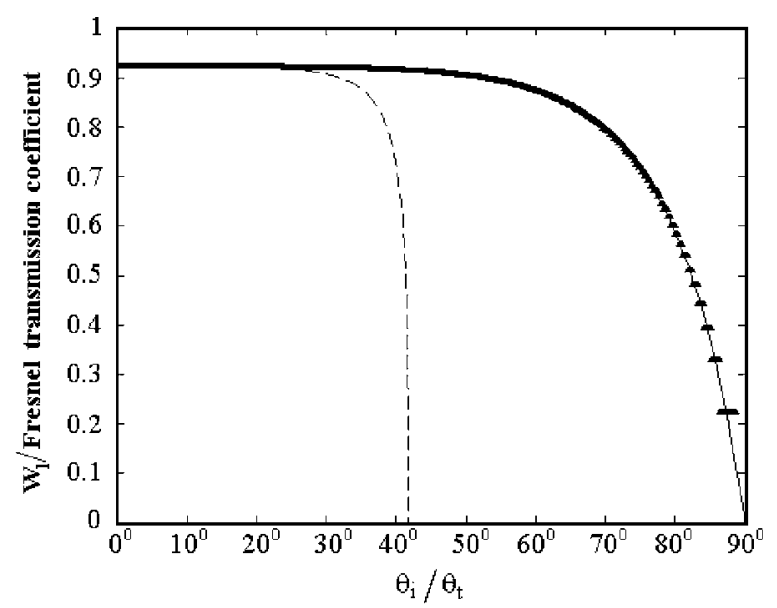

Figure 5. The Fresnel transmission coefficients for the light radiation emergent from the stronger $n=1.5$ to the less optically dense medium $n_{0}=1.0$. The triangles $(\Delta)$ present the MC results of $W_{1}$, the angular weight distribution. The solid line $(-)$ is the same dependence on the exit angle $\theta_{t}$ (see figure 4). The dashed line presents the Fresnel transmission coefficient (dependence on the angle of incidence $\theta_{i}$ ).

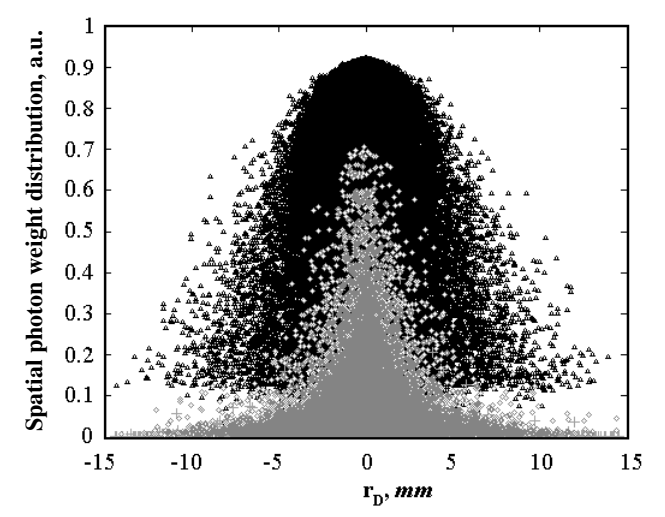

(a)

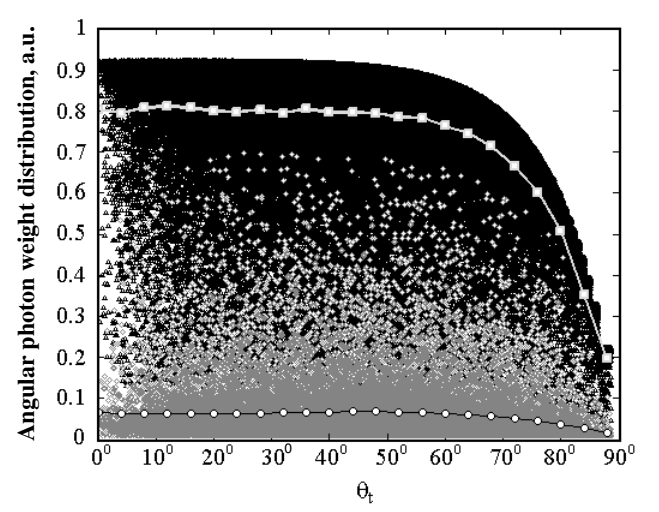

(b)

Figure 6. The photon packet weight distribution on the detector area $\left(\boldsymbol{r}_{\mathrm{D}}\right)$ in the presence of the medium absorption $\left(\mu_{a}=0.01 \mathrm{~mm}^{-1}\right)$ : (a) spatial photon weight distribution versus the detector area diameter; (b) angular photon weight distribution over the exit angle $\theta_{t}$. The triangles $(\Delta)$ specify the weight of the photon packets free of reflection/refraction on the medium boundary, and open square $(\square)$ show their average angular weight distribution. The diamonds $(\diamond)$ represent the weight of the photon packets having reflected/refracted at the boundary twice, circles $(O)$ are the average angular weight of these photon packets.

Fresnel's reflection and transmission coefficients (7) were also pre-calculated for the discrete number of incident angles $\left(\cos ^{-1}(0.001)\right)$. This gives a sufficient reduction in the calculation time.

An introduction of the absorption into the model distorts the discreteness in both the spatial and angular photon weight distributions (figure 6). However, it does not dramatically affect the behaviour of average angular weight distributions (see figure 6(b)). It should be pointed out, that here (see figures 6(a) and (b)) we represent the photon weight distributions 


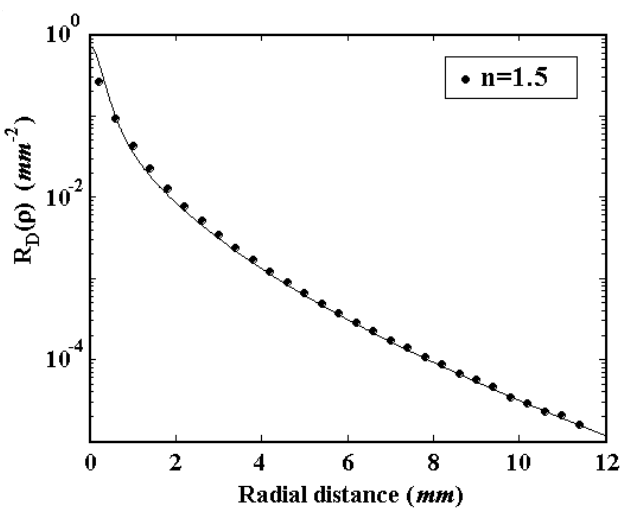

Figure 7. Comparison of the results from the diffusion theory and MC simulations for spatially resolved reflectance from the index-mismatched interface of the semi-infinite medium. The solid line is the prediction of the diffusion theory (Kienle and Patterson 1997). The symbols are the MC simulation results: the solid circles $(\bullet)$ are for the index-mismatched interface: the relative refractive index $n=1.5$. The optical parameters are: $\mu_{s}=30 \mathrm{~mm}^{-1}, \mu_{a}=0.01 \mathrm{~mm}^{-1}$ and $g=0.9$.

similar to those described above (see figures 3(a) and (b)), but in the presence of medium absorption $\left(\mu_{a}=0.01 \mathrm{~mm}^{-1}\right)$.

Despite the discreteness of the photon weight distribution, the distribution of the radiation intensity detected at the medium boundary, defined as

$$
I\left(\boldsymbol{r}_{\mathrm{D}}\right)=\int W\left(\boldsymbol{r}_{\mathrm{D}}\right) \mathrm{d} \boldsymbol{r}_{\mathrm{D}}
$$

exhibits the coordinate dependence predicted by the diffusion theory (see figure 7). Here, $r_{\mathrm{D}}$ is the distance from the source origin to the detector area, where the photon packets are registered, $W\left(\boldsymbol{r}_{\mathrm{D}}\right)$ is the total photon packets weight at the detector (see equation (8)).

\subsection{Spatial photon sensitivity profiles}

We define the spatial photon sensitivity profile as the probability density function of the photon medium sampling (Okada et al 1997, Meglinskii and Matcher 2001b):

$$
Q(\boldsymbol{r})=\frac{\sum_{j=1}^{N} \sum_{i=1}^{F_{j}} U_{i}(\boldsymbol{r}) W_{j}}{\sum_{j=1}^{N} W_{j}} .
$$

Here $W_{j}$ is the final weight of the $j$ th photon packet, $N$ is the total number of the detected photon packets, $F_{j}$ is the number of scattering events experienced by the $j$ th photon packet on its way from the source to detector, $U_{i}(r)$ is the function determining the existence of the $j$ th photon packet at a point $\boldsymbol{r}$ in the medium. To calculate $U_{i}(\boldsymbol{r}) W_{j}$ we divide the medium by the cubic cells $10 \mu \mathrm{m} \times 10 \mu \mathrm{m} \times 10 \mu \mathrm{m}$. Then the photon packet weights $W_{j}$ are accumulated in the cells having the photon packet trajectories.

The results of the SPSP calculation for a randomly inhomogeneous, highly scattering and absorbing semi-infinite medium are shown in figure 8 . The optical properties of the medium are: $\mu_{s}=30 \mathrm{~mm}^{-1}, \mu_{a}=0.01 \mathrm{~mm}^{-1}, g=0.9$. Two refractive indices that are considered are $n=$ 1.5 and $n=1.05$, respectively. The refractive index of the ambient space $n_{0}$ is 1 . The SPSPs are calculated for a geometry in which the source and the detector areas (both $100 \mu \mathrm{m}$ in diameter) 


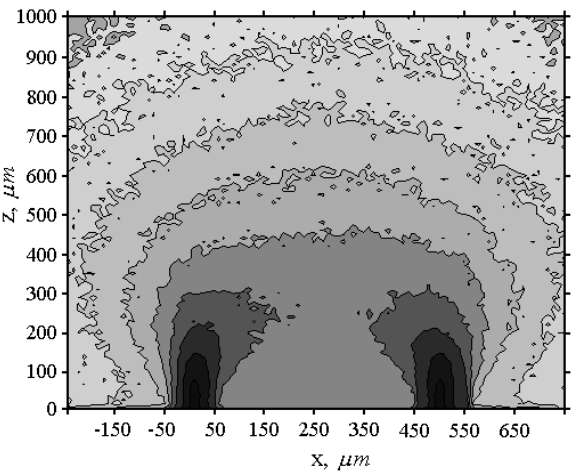

(a)

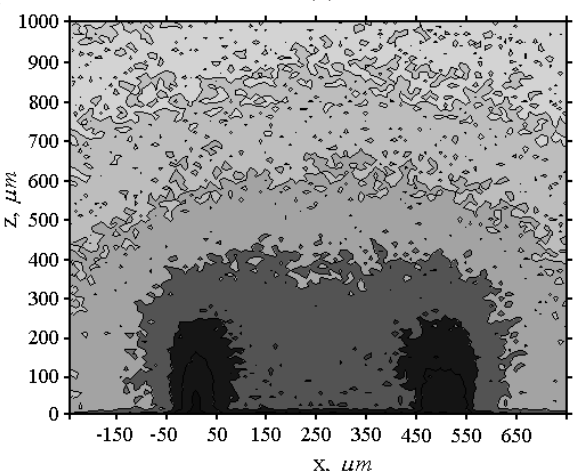

(c)

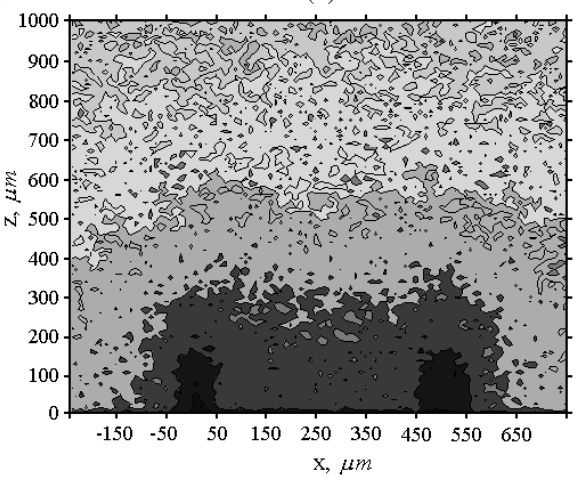

(e)

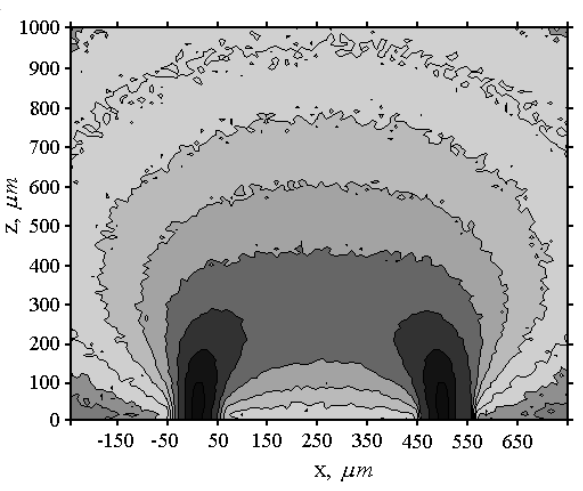

(b)

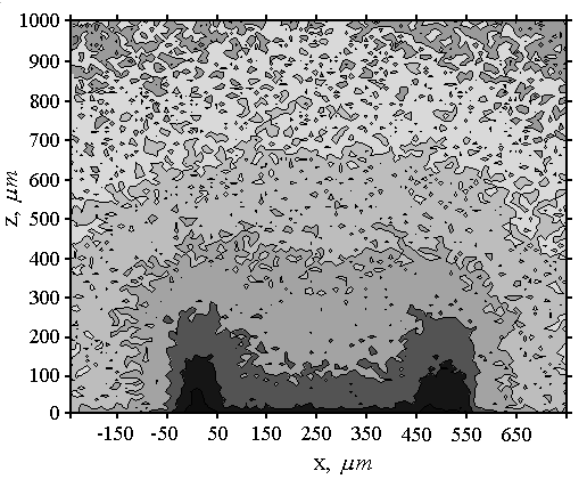

(d)

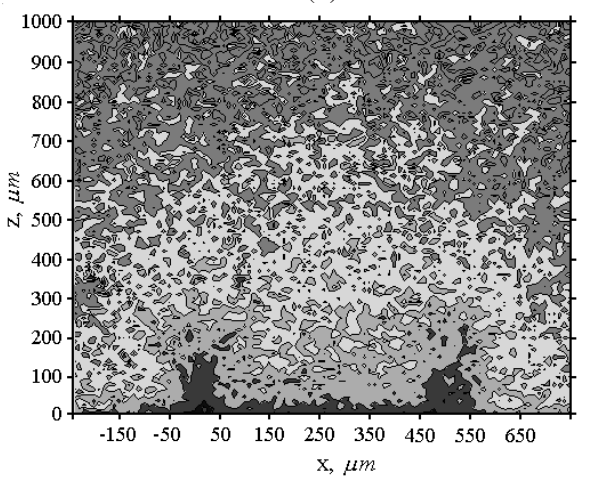

(f)

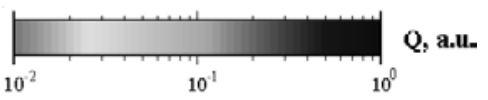

Figure 8. $X O Z$ plane of spatial photon sensitivity profiles for: $W_{1}$ photon packets, detected free of reflections/refractions on the boundary of a medium with refractive indexes 1.5 (a) and 1.05 (b); (c) and (d) show SPSPs for the $W_{2}$ photon packets experienced by one reflection/refraction on the medium surface before the detection, in cases when the refractive index of medium is 1.5 and 1.05, respectively; (e) and (f) represent SPSPs for $W_{3}$ high order interactions of the photon packets within the medium surface, for the 1.5 and 1.05 medium refractive indexes, respectively. Other optical properties of modelling medium are: $\mu_{s}=30 \mathrm{~mm}^{-1}, \mu_{a}=0.01 \mathrm{~mm}^{-1}$ and $g=0.9$. Centre-to-centre source-detector spacing is $500 \mu \mathrm{m}$, and the diameters of both the source and the detector are $100 \mu \mathrm{m}$. 


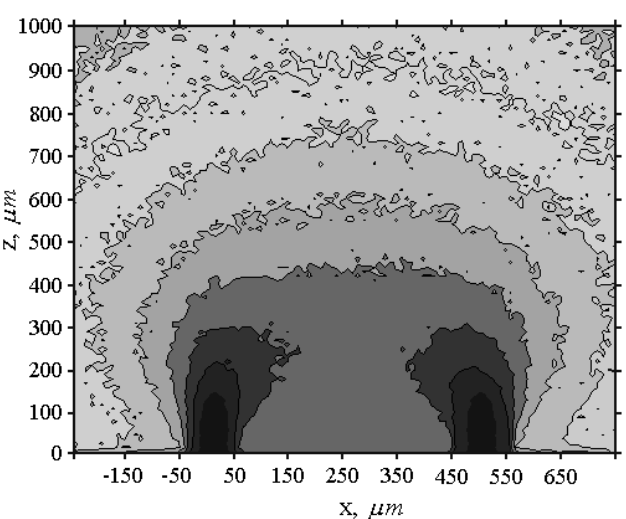

(a)

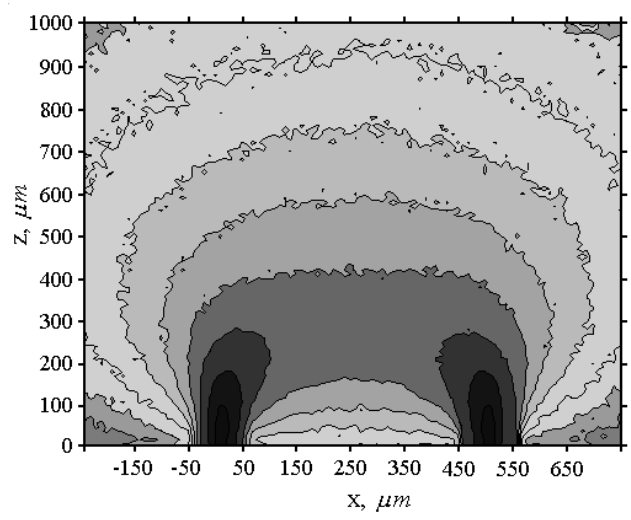

(c)

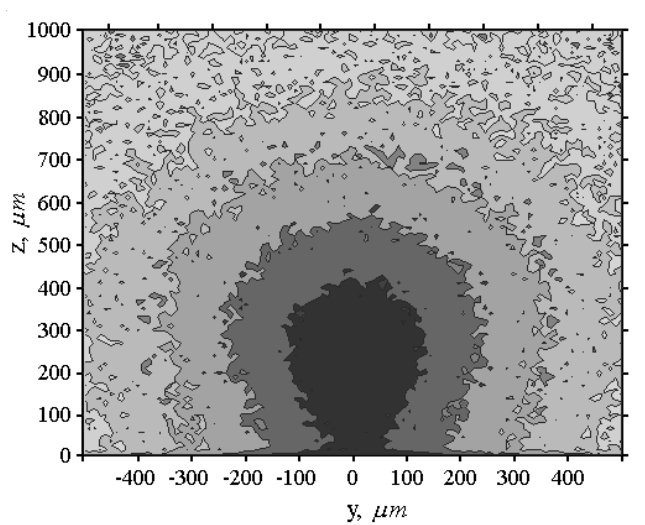

(b)

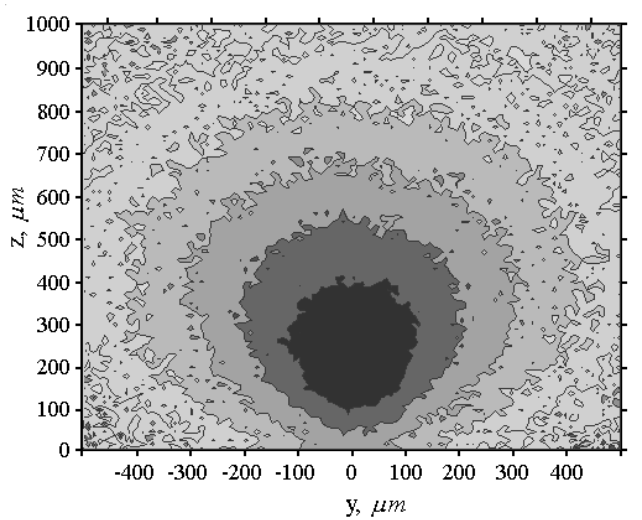

(d)

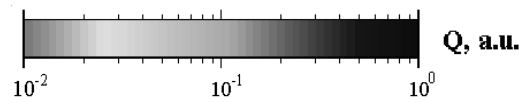

Figure 9. 2D spatial photon sensitivity profiles: $X O Z$ planes (a) and (c), and $Y O Z$ plane midway between the source-detector (b) and (d). SPSPs are calculated for a randomly inhomogeneous semi-infinite highly scattering absorbing medium with the refractive index $n=1.5$ for (a) and (b), and $n=1.05$ for (c) and (d). Other optical properties are: $\mu_{s}=30 \mathrm{~mm}^{-1}, \mu_{a}=0.01 \mathrm{~mm}^{-1}$, and $g=0.9$. Both source and detector diameters are $100 \mu \mathrm{m}$, and source-detector spacing is $500 \mu \mathrm{m}$ centre-to-centre.

are separated centre-to-centre by $500 \mu \mathrm{m}$. The greyscale bar based on the power of 10 shows the ratio of the photon weight distributions.

Let us consider the SPSPs from the viewpoint of $W_{1}, W_{2}, W_{3}$ photon packet weight distributions. The $W_{1}$ photon weight distribution (see figure $8(\mathrm{a})$ ) is close, both quantitatively and qualitatively, to the total SPSP (see figure 9 (a)), whereas due to multiple scattering and back reflection on the medium boundary, the distributions of the $W_{2}$ and $W_{3}$ fractions have the shapes which are different from $W_{1}$. Figures 8(a), (c) and (e) and (b), (d) and (f) illustrate the results of the SPSP simulation for the two refractive indices, 1.5 and 1.05, respectively. It is easy to see that $W_{1}$ photon packets (photon packets free of pre-detecting internal reflections/refractions at the medium boundary) localize deeper into the medium 


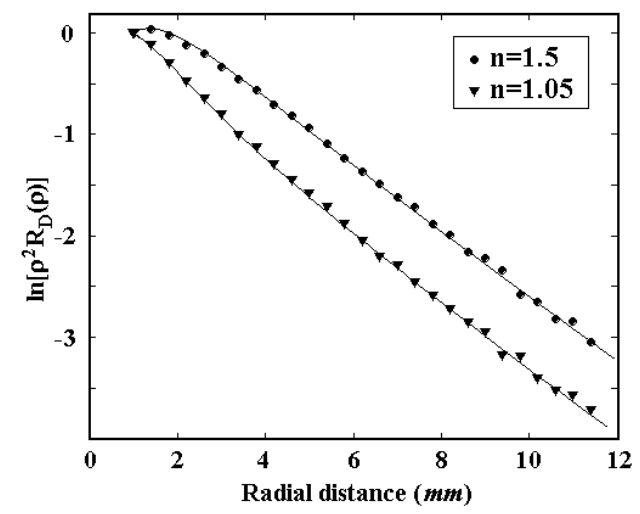

Figure 10. Spatially resolved diffuse reflectance from a semi-infinite highly scattering medium is plotted as log of the reflectance multiplied by the square of the radial distance (Farrell and Patterson 2001). The symbols show the results of the MC simulation, and the solid lines are the results of the diffusion theory prediction. The optical parameters are: $\mu_{s}=30 \mathrm{~mm}^{-1}, \mu_{a}=0.01 \mathrm{~mm}^{-1}$, $g=0.9, n=1.5$ (circles) and $n=1.05$ (triangles).

(see figure $8(\mathrm{a})$ ), whereas $W_{2}$ and $W_{3}$ photon packets mainly characterize the topical regions of the medium (see figures $8(\mathrm{c})$ and (e)).

It is logically presumed then that matching of refractive indices of the modelled medium and ambient space increases the number of $W_{1}$ photon packets and restricts their $W_{2}$ and $W_{3}$ fractions. In fact, the results of simulation with the reduced medium refractive index $(n=$ 1.05) (figures $8(\mathrm{~b}),(\mathrm{d})$ and (f)) suggest that the $W_{2}$ and $W_{3}$ fractions of the photon packets are restricted in number, and tend to spatially localize in a topical region of the medium (see figures 8(d) and (f)). Moreover, it is significant that refractive index match affects the contrast of the SPSP images (see figures 8(b), (d) and (f)) in comparison to the case of the refractive index mismatch (see figures $8(\mathrm{a}),(\mathrm{c})$ and (e)).

The total SPSPs, calculated as the sum of the $W_{1}, W_{2}$ and $W_{3}$ photon packet fractions, are shown in figure 9. These graphs give an idea as to how to detect sensitivity spreads between the source and the detector, and which deepest region of the medium is being sampled. It also shows that the localization of the SPSP is significantly improved in the case of refractive index match, and the highly sensitive region is deflected from the medium surface (see figure 9(c) compared with figure 9(a), and figure 9(b) compared with figure 9(d), respectively). In figure 9 the contour plots are normalized by a maximum value of the XOZ image. As expected, the SPSP resembles the so-called 'banana' shape (see figures 9(a) and (c)) which means that it is in good agreement with the results previously carried out in researches (Feng et al 1993, Okada et al 1997, Okada 2000, Meglinsky and Matcher 2001a). The contours of the SPSP plots for both the mismatched and matched refractive index cases cover roughly the same depth of signal localization (see figure 9). These SPSPs, presented in figures 9(a) and (c), and their cross sections (see figures 9(b) and (d)) are calculated with an equal number of photon packets.

We emphasize that the results of the spatially resolved diffuse reflectance simulation obtained by the presented MC technique directly agree while the results of the improved steady-state diffusion equation (Kienle and Patterson 1997) and with the experimental results carried out by Farrell and Patterson (2001). To illustrate this in figure 10 the spatially resolved reflectance $\ln \left[\rho^{2} R_{\mathrm{D}}(\rho)\right]$ is plotted against $\rho$ in a similar way to Farrell and Patterson (2001). $R_{\mathrm{D}}(\rho)$ is the steady-state diffuse reflectance per incident photon (Haskell et al 1994, 
Kienle and Patterson 1997):

$$
R_{\mathrm{D}}(\rho)=\int_{2 \pi} \mathrm{d} \Omega[1-R(\theta)] \frac{1}{4 \pi}\left[\Phi(\rho)+3 D \frac{\partial \Phi(\rho)}{\partial z} \cos \theta\right] \cos \theta
$$

where $\Phi(\rho)$ is the fluence rate at the medium surface, $\rho$ is the radial distance from the source to the point of detection and $D$ is the diffusion constant.

\section{Conclusions}

The model calculations were carried out with the aim of studying and verifying the effect of the refractive index match at the medium boundary and its influence on the diffuse reflectance. The MC technique is used for the simulation of SPSP and photon migration through a 3D randomly inhomogeneous, semi-infinite highly scattering and absorbing medium limited by a plane boundary. The internal reflection on the surface of the medium has been taken into account by splitting the trajectories of the photon packets on the medium boundary into reflected and transmitted parts. The statistical weights of these parts are weakened according to Fresnel's reflection/transmission coefficient. The chosen optical properties of the modelling medium are close to the optical properties typical of biological tissues.

The results of the SPSP simulation (see figure 9) give a graphical perception of the detected signal distribution both at the detector area and through the randomly inhomogeneous, semiinfinite highly scattering medium. The results demonstrate that the matching of the refractive index of the medium significantly improves the contrast and spatial resolution of the SPSP. Whereas reduction of the scattering increases permeation of light into the medium. This elucidates the influence of refractive index matching on the diffuse reflectance.

The effect of the refractive index match is predicted correctly by the MC method and diffusion approximation. This is illustrated by the results of simulation of the spatially resolved diffuse reflectance (see figure 10) which agree well with the results predicted by the diffusion approximation (Kienle and Patterson 1997), and the experimental results (Farrell and Patterson 2001).

We believe that the present results are important from the viewpoint of image contrast and spatial resolution, as well as its mechanism. These modelling results are a brief survey of the potential of the modelling study of the effects of refractive index match. At present more complete studies are under way to demonstrate the influence of the refractive index matching at the image reconstruction of an inhomogeneity embedded in the medium.

\section{Acknowledgments}

The authors thank Dr S J Matcher of the University of Exeter (Exeter, UK) and Dr M C Jermy of the Cranfield University (Cranfield, UK) for useful discussions during this study. DYC also acknowledges the support of the Department of Optical and Automotive Engineering, School of Engineering, Cranfield University.

\section{References}

Arridge S R 1995 Photon-measurement density functions: Part 1. Analytical forms Appl. Opt. 34 7395-409

Bonner R F, Nossal R, Havlin S and Weiss G H 1987 Model for photon migration in turbid biological media J. Opt. Soc. Am. A 4 423-32

Chernomordik V, Gandjbakhche A H, Hebden J C and Zaccanti G 1999 Effect of lateral boundaries on contrast functions in time-resolved transillumination measurements Med. Phys. 26 1822-31 
Farrell T J and Patterson M S 2001 Experimental verification of the effect of refractive index mismatch on the light fluence in a turbid medium J. Biomed. Opt. 6 468-73

Feng S, Fanan Z and Chance B 1993 Monte Carlo simulations of photon migration path distributions in multiple scattering media Proc. SPIE $188878-89$

Flock S T, Patterson M S, Wilson B C and Wyman D R 1989 Monte Carlo modeling of light propagation in highly scattering tissue: 1. Model predictions and comparison with diffusion theory IEEE Trans. Biomed. Eng. 36 $1162-8$

Gandjbakhche A H and Weiss G H 1995 Progress in Optics vol 34 ed E Wolf (Amsterdam: North-Holland) pp 333-401

Haskell R C, Svaasand L O, Tsay T T, Feng T C, McAdams M S and Tromberg B J 1994 Boundary conditions for the diffusion equation in radiative transfer J. Opt. Soc. Am. A $112727-41$

Henyey L G and Greenstein J L 1941 Diffuse radiation in the galaxy Astrophys. J. 93 70-83

Hiraoka M, Firbank M, Essenpreis M, Cope M, Arridgem S R, van der Zee P and Delpy D T 1993 A Monte Carlo investigation of optical pathlength in inhomogeneous tissue and its application to near-infrared spectroscopy Phys. Med. Biol. 38 1859-76

Ishimaru A 1978 Wave Propagation and Scattering in Random Media (New York: Academic) p 572

Jermy M C and Allen A 2001 Multiple scattering in dense spray images: Monte Carlo simulation 6th Int. Congress in Optical Particle Characterization (Brighton, 2-5 April)

Jermy M C and Allen A 2002 Simulation the effects of multiple scattering on images of dense sprays and particle fields Appl. Opt. 41 4188-96

Keijzer M, Jacques S L, Prahl S A and Welch A J 1989 Light distributions in artery tissue: Monte Carlo simulations for finite-diameter laser beams Laser Surg. Med. 9 148-54

Kienle A, Lilge L, Patterson M S, Hibst R, Steiner R and Wilson B C 1996 Spatially resolved absolute diffuse reflectance measurements for noninvasive determination of the optical scattering and absorption coefficients of biological tissue Appl. Opt. 35 2304-14

Kienle A and Patterson M S 1997 Improved solutions of the steady-state and the time-resolved diffusion equations for reflectance from semi-infinite turbid medium J. Opt. Soc. Am. A 14 246-54

Lagendijk A, Vreeker R and de Vries P 1989 Influence of internal reflection on diffusive transport in strongly scattering media Phys. Lett. A 136 81-8

Lux I and Koblinger L 1991 Monte Carlo Particle Transport Methods: Neutron and Photon Calculations (Boca Raton, FL: CRC Press) p 517

Matcher S J 2002 Signal quantification and localization in tissue near-infrared spectroscopy Handbook of Optical Biomedical Diagnostics ed V V Tuchin (Washington, DC: SPIE-The International Society for Optical Engineering) pp 487-584

Meier R P, Lee J S and Anderson D E 1978 Atmospheric scattering of middle UV radiation from an internal source Appl. Opt. 17 3216-25

Meglinsky I V and Matcher S J 2001a Modelling the sampling volume for skin blood oxygenation measurements Med. Biol. Eng. Comput. 39 44-50

Meglinskii I V and Matcher S J 2001b Analysis of the spatial distribution of the detector sensitivity in a multilayer randomly inhomogeneous medium with strong light scattering and absorption by the Monte Carlo method Opt. Spectrosc. $91654-9$

Meglinski I V and Matcher S J 2002 Quantitative assessment of skin layers absorption and skin reflectance spectra simulation in the visible and near infrared spectral regions Physiol. Meas. 23 741-53

Meglinski I V, Matcher S J, Bashkatov A N, Genina E A and Tuchin V V 2001 Confocal probing of skin during it clearing IEEE Proc. CLEO/Pacific Rim 1 I234-5

Mourant J R, Bigio I J, Jack D A, Johnson T M and Miller H D 1997 Measuring absorption coefficients in small volumes of highly scattering media: source-detector separations for which path lengths do not depend on scattering properties Appl. Opt. 36 5655-61

Nossal R, Keifer J, Weiss G H, Bonner R F, Taitelbaum H and Havlin S 1988 Photon migration in layered media Appl. Opt. 27 3382-91

Okada E 2000 The effects of superficial tissue of the head on spatial sensitivity profiles for near infrared spectroscopy and imaging Opt. Rev. $7375-82$

Okada E, Firbank M, Schweiger M, Arridge S R, Cope M and Delpy D T 1997 Theoretical and experimental investigation of near-infrared propagation in a model of the adult head Appl. Opt. 36 21-31

Prahl S A, Keijzer M, Jacques S L and Welch A J 1989 A Monte Carlo model of light propagation in tissue SPIE Inst. Ser. IS 5 102-11

Ripoll J and Nieto-Vesperinas M 1999 Index mismatch for diffuse photon density waves at both flat and rough diffuse-diffuse interfaces J. Opt. Soc. Am. A 16 1947-57

Sobol' I M 1975 The Monte Carlo Method (Chicago: University of Chicago Press) p 65 
Star W 1995 Diffusion theory of light transport Optical-Thermal Response of Laser-Irradiated Tissue ed A J Welch and M J C van Gemert (New York: Plenum) pp 131-206

Thomas G E and Stamnes K 1999 Radiative Transfer in the Atmosphere and Ocean (Cambridge: Cambridge University Press) p 546

Tuchin V 2000 Tissue Optics: Light Scattering Methods and Instruments for Medical Diagnosis (Washington, DC: SPIE-The International Society for Optical Engineering) p 353

Tuchin V V 2002 Handbook of Optical BioMedical Diagnostics (Washington, DC: SPIE_The International Society for Optical Engineering) p 1110

Tuchin V V, Maksimova I L, Zimnyakov D A, Kon I L, Mavlutov A H and Mishin A A 1997 Light propagation in tissues with controlled optical properties J. BioMed. Opt. 2 401-17

Vargas G, Chan K F, Barton J K, Rylander H G and Welch A J 1999 Use of a an agent to reduce scattering in skin Laser Surg. Med. 24 133-41

Vargas G, Chan K F, Tomsen S L and Welch A J 2001 Use of osmotically active agents to alter optical properties of tissue: effects on the detected fluorescence signal measured through skin Laser Surg. Med. 29 213-20

Wang L, Jacques S L and Zheng L 1995 MCML_-Monte Carlo modelling of light transport in multi-layered tissues Comput. Meth. Progr. Biomed. 47 131-46

Wang R K, Xu X, Tuchin V V and Elder J B 2001 Concurrent enhancement of imaging depth and contrast for optical coherence tomography by hyperosmotic agents J. Opt. Soc. Am. B $18948-53$

Wiersma D S, Bartolini P, Lagendijk A and Righini K 1997 Localization of light in a disordered medium Nature 390 671-3

Wilson B C and Adam G 1983 A Monte Carlo model for absorption and flux distributions of light in tissue Med. Phys. 10 824-30

Witt A N 1977 Multiple scattering in reflection nebulae: 1. Monte Carlo approach Astrophys. J. 35 1-6

Yaroslavsky I V and Tuchin V V 1992 Light transport in multilayed scattering media. Monte Carlo modelling Opt. Spectrosc. 72 934-9

Zhu J X, Pine D J and Weitz DA 1991 Internal reflection of diffusive light in random media Phys. Rev. A 44 3948-59 